International Europhysics Conference on High Energy Physics, Marseille, July 22-28, 1993, parallel session : Neutrino Physics and non-accelerator experiments.

\title{
FIRST RESULTS OF A SEARCH FOR DOUBLE BETA DECAY OF 100Mo WITH THE NEMO 2 DETECTOR
}

\section{NEMO Collaboration}

R. Amold 6 , A. Barabash 4 , A. V. Blinov 1 , D. Blum 5 , V. Brudanin 7 , J.E. Campagne, F. Danevich ${ }^{3}$, D. Dassié ${ }^{1}$, V. Egorov 7 , J.L. Guyonnet ${ }^{6}$, F. Hubert ${ }^{1}$, Ph. Hubert ${ }^{1}$, M. C. Isaac ${ }^{1}$, C. Izac'1, S. Jullian ${ }^{5}$, O. Kochetov 7 , V. Kouts ${ }^{3}$, D. Lalanne ${ }^{5}$, T. Lamhamdi6, F. Laplanche ${ }^{5}$, F. Leccia ${ }^{1}$, Yu. Lepichin ${ }^{4}$, I. Linck 6 , C. Longuemare 2 , F. Mauger ${ }^{2}$, P. Mennrath ${ }^{1}$, F. Natchez ${ }^{5}$, H.W. Nicholson 8 , A. Nozdrin 7 , G. Pichenot ${ }^{5}$, F. Piquemal 6 , F. Scheibling ${ }^{6}$, C.S. Sutton ${ }^{8}$, G. Szklarz ${ }^{5}$, V. Tretyak ${ }^{3}$, V. Umatov ${ }^{4}$, I. Vanushin ${ }^{4}$, Yu. Vassilyev5, Ts. Vylov7 , Yu. Zdesenko ${ }^{3}$, Sh. Zaparov7.

1. Centre d'Erudes Nucléaires, I N2 P3- CNRS et Université de Bordeaux, 33170 Gradignan, France

2. Laboratoire de Physique Corpusculaire, I N2 P3-CNRS et Universite de Caen, 14032 Caen, France

3. Institute for Nuclear Research of the Ukrainian Academy of Sciences, Kiev, Ukraine

4. Institute for Theoretical and Experimental Physics, ITEP, Moscow, Russia

5. Laboratoire de l'Accélérateur Linéaire, I N2 P3-CNRS et Université de Paris-Sud, 91405 Orsay, France

6. Centre de Recherches Nucléaires, I N2 P3-CNRS et Université Louis Pasteur, 67037 Strasbourg, France

7. Joint Institute for Nuclear Research, Dubna, Russia

8. Mount Holyoke College, South Hadley, MA 01075, U.S.A.

$$
\text { FGO2 - } 90 \text { EK } 40553
$$


Double beta decay of $100 \mathrm{Mo}(172 \mathrm{~g})$ is studied with the NEMO 2 detector in the Fréjus Underground Laboratory. The experiment has now accumulated 2485 hours of data taking. A clear signal of 380 events for $2 \beta 2 v$ decay has been obtained corresponding to a half-life of $T_{1 / 2}=1.0 \pm 0.08$ (stat.) \pm 0.2 (syst.) $10^{19} \mathrm{y}$. Limits are presented for $2 \beta(0 \nu, \chi), 2 \beta 0 v$ (ground state and excited states $2_{1}^{+}$and $0_{1}^{+}$). The experiment will run till October 1993.

\section{INTRODUCTION}

Neutrinoless double beta decay experiments $(2 \beta 0 \mathrm{v}), \mathrm{A}(\mathrm{N}, \mathrm{Z})--->\mathrm{A}(\mathrm{N}-2, \mathrm{Z}+2)$ $+2 e^{-}$, are considered an important test of the Standard Electroweak Model. The observation of such a process would imply Majorana neutrinos with non-zero masses and also possible right handed current contribution in the weak leptonic interaction. In the same kind of experiments the existence of a Majoron can also be investigated with the process $2 \beta(0 v, \chi), A(N, Z)-->A(N-2, Z+2)+2 e^{-}+\chi$. The double beta two neutrino decay $(2 \beta 2 v), A(N, Z) \cdots A(N-2, Z+2)+2 e^{-}+2 v$, corresponds to a second order Fermi process and is measured in the present experiment with the isotope $100 \mathrm{Mo}$.

The NEMO collaboration plans to build an experiment to study with $10 \mathrm{~kg}$ of $>95 \%$ enriched $100 \mathrm{Mo} 2 \beta 0 \mathrm{v}$ decay. The experiment is designed to probe the effective Majorana neutrino mass down to $0.1 \mathrm{eV}$. Since four years the NEMO collaboration has been involved in a Research and Development program and has built two prototypes NEMO 1 and NEMO 2 (Ref 1,2 ) to measure the experimental e-e background in the $3 \mathrm{MeV}$ region $\left(\mathrm{Q}=3.03 \mathrm{MeV}\right.$ for the $\left.{ }^{100 \mathrm{Mo}} 2 \beta 0 \mathrm{~V}\right)$.

The detector NEMO 2 (Ref. 1) operates in the Fréjus Underground Laboratory (4800 m.w.e depth) and has been running since August 91. Two experimental runs to study backgrounds have been completed and a third run starting in December 92 provides the data presented here which was taken with a source of enriched $100 \mathrm{Mo}$.

\section{THE NEMO 2 SET-UP}

The NEMO 2 (Fig.1) detector consists of a source foil $(1 \mathrm{~m} * 1 \mathrm{~m} * 40 \mu \mathrm{m})$ which splits the $1 \mathrm{~m}^{3}$ tracking volume in two parts. The tracking device is formed by 10 frames of $2 * 32$ Geiger cells identical to those of the previous detector NEMO 1 (Ref. 2). A frame is made of twc crossed planes of cells orientated vertically and horizontally. The tracking volume is filled with Helium gas with $2 \%$ ethyl alcohol and is enclosed inside sheets of aluminized Mylar $(36 \mu \mathrm{m})$. On each side of the tracking 


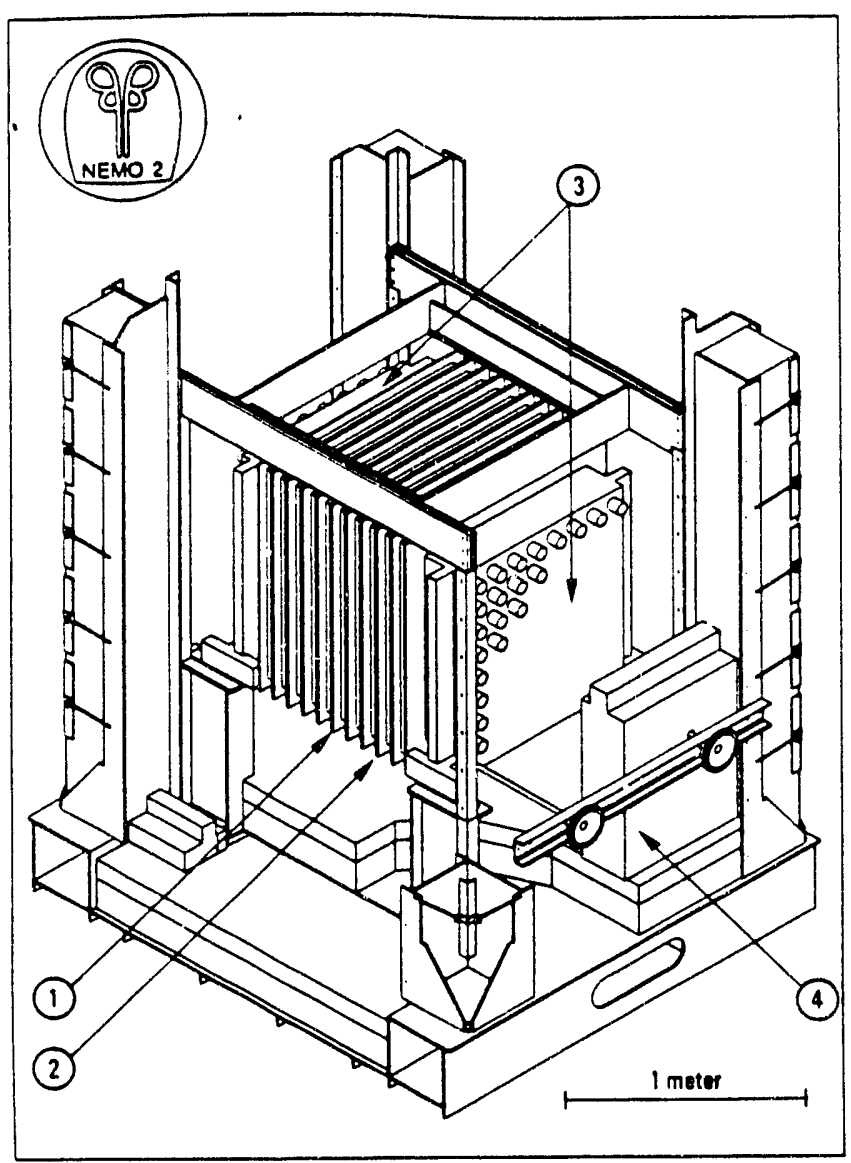

Fig. 1

The NEMO 2 detector.

1 - central frame containing the source foils

2 - frames of Geiger cells (total number of cells 640 )

3 - scintillator walls with associated PMTs $(2 * 64$ PMT"s)

4 - part of the shielding $(20 \mathrm{~cm}$ iron $+5 \mathrm{~cm}$ lead)

volume is an array of 64 phoswich counters. Each one $(12 \mathrm{~cm} * 12 \mathrm{~cm})$ is made of $2 \mathrm{~cm}$ of plastic scintillator and $2.5 \mathrm{~mm}$ of $\mathrm{CsI}(\mathrm{Na})$ in thickness. These two walls are devoted to energy and time of flight measurements. The detection threshold is $50 \mathrm{keV}$ for an electron and the energy distribution shows a FWHM of $16 \%$ at $1 \mathrm{MeV}$. The time of flight standard deviation is measured to be $\sigma=300$ ps for $E=$ $500 \mathrm{keV}$ for one counter. Taking into account the absorption before the CsI layer, the computed efficiency of detection of an X-ray inside the CsI is $70 \%$ at $80 \mathrm{keV}$. The detection efficiency of a $\gamma$-ray is $35 \%$ at $500 \mathrm{keV}$ in the phoswich. Delayed tracks are recorded up to $1 \mathrm{~ms}$ after each event in order to identify $\alpha$ particles emitted by ${ }^{214} \mathrm{Bi}$ in the case of internal pollution.

This active detector is shielded inside iron $(20 \mathrm{~cm})$ and lead $(5 \mathrm{~cm})$. All the materials used in the detector have been carefully chosen for their low activity (Ref. 3) with the exception of the photomultiplier tubes. Normal Radon activity in the Laboratory during data collection is of the order of $30 \mathrm{~Bq} / \mathrm{m}^{3}$.

The current source foil is divided into two parts, one is made of enriched $100 \mathrm{Mo}$.(fiducial surface $X=\{-47.5 \mathrm{~cm}, 2.5 \mathrm{~cm}\}, Y=\{-46 \mathrm{~cm}, 46 \mathrm{~cm}\}$, fiducial mass $=172 \mathrm{~g}$ ), the other part with natural Molybdenum (fiducial surface $X=\{4.5 \mathrm{~cm}$, $47.5 \mathrm{~cm}\}, Y=\{-46 \mathrm{~cm}, 46 \mathrm{~cm}\}$, fiducial mass $=163 \mathrm{~g})$. 
Low gamma activity measurements of the foils were made with a low background Germanium detector in the Fréjus Underground Laboratory. The corresponding limits are

$<2 \mathrm{dpm} / \mathrm{kg}$ of $234 \mathrm{Th}$ and $234 \mathrm{mPa}$,

$<2 \mathrm{dpm} / \mathrm{kg}$ of $214 \mathrm{Bi}$,

$<0.3 \mathrm{dpm} / \mathrm{kg}$ of $208 \mathrm{Tl}$

$(\mathrm{dpm} / \mathrm{kg}=$ decay per minute per kilogram).

\section{DATA ACQUISITION AND EVENT SELECTION}

The trigger used to study double beta decay requires the firing of at least 2 plastic counters (threshold $50 \mathrm{keV}$ ) within 50 ns followed by 4 Geiger modules within $2.5 \mu \mathrm{s}$. The trigger rate of $0.23 \mathrm{~Hz}$ has shown good stability.

As examples, four distinct types of events are shown in Fig. 2 which are clearly identified in the NEMO 2 detector. An electron is defined by a non-scattering track starting in the foil and reaching one scintillator with an energy deposit greater than $200 \mathrm{keV}$ to minimize time of flight corrections. A $\gamma$-ray signature is defined by one or two adjacent scintillators fired (energy deposition in at least one of the scintillators has to be greater than $200 \mathrm{keV}$ ). An X-ray corresponds to an isolated CsI fired.

A track reconstruction program was used to select events. The vertex position in the source plane is known to the order of $1 \mathrm{~cm}$ (FWHM) limited by multiple scattering in the Helium gas.

\section{SIMULATIONS}

In order to analyse the data of NEMO 2, simulations have been carried out. The GEANT code was used and tuned for electron energies down to the threshold used in the analysis. Several potential sources of internal background have been studied. In Table 1 efficiencies are given for the most troublesome nuclei for $2 \beta 2 \mathrm{v}$ background and those able to produce events with an energy deposit greater than $2 \mathrm{MeV}$. In addition, efficiencies for e- $\gamma$ channels are given (Ref. 4).

\begin{tabular}{|c|c|c|c|c|}
\hline$\varepsilon$ & ${ }^{208} \mathrm{Tl}$ & ${ }^{214} \mathrm{Bi}$ & ${ }^{228} \mathrm{Ac}$ & $234 \mathrm{~m}_{\mathrm{Pa}}$ \\
\hline $\mathrm{e}-\mathrm{e}\left(\times 10^{-4}\right)$ & 12.2 & 7.0 & 3.0 & 3.2 \\
$\mathrm{e}-\gamma\left(\times 10^{-3}\right)$ & 7.8 & 4.3 & 2.0 & $<0.1$ \\
\hline
\end{tabular}

Table 1: Efficiencies for nuclei contributing to $2 \beta 2 v$ background and those able to produce events with an energy deposit greater than $2 \mathrm{MeV}$. 


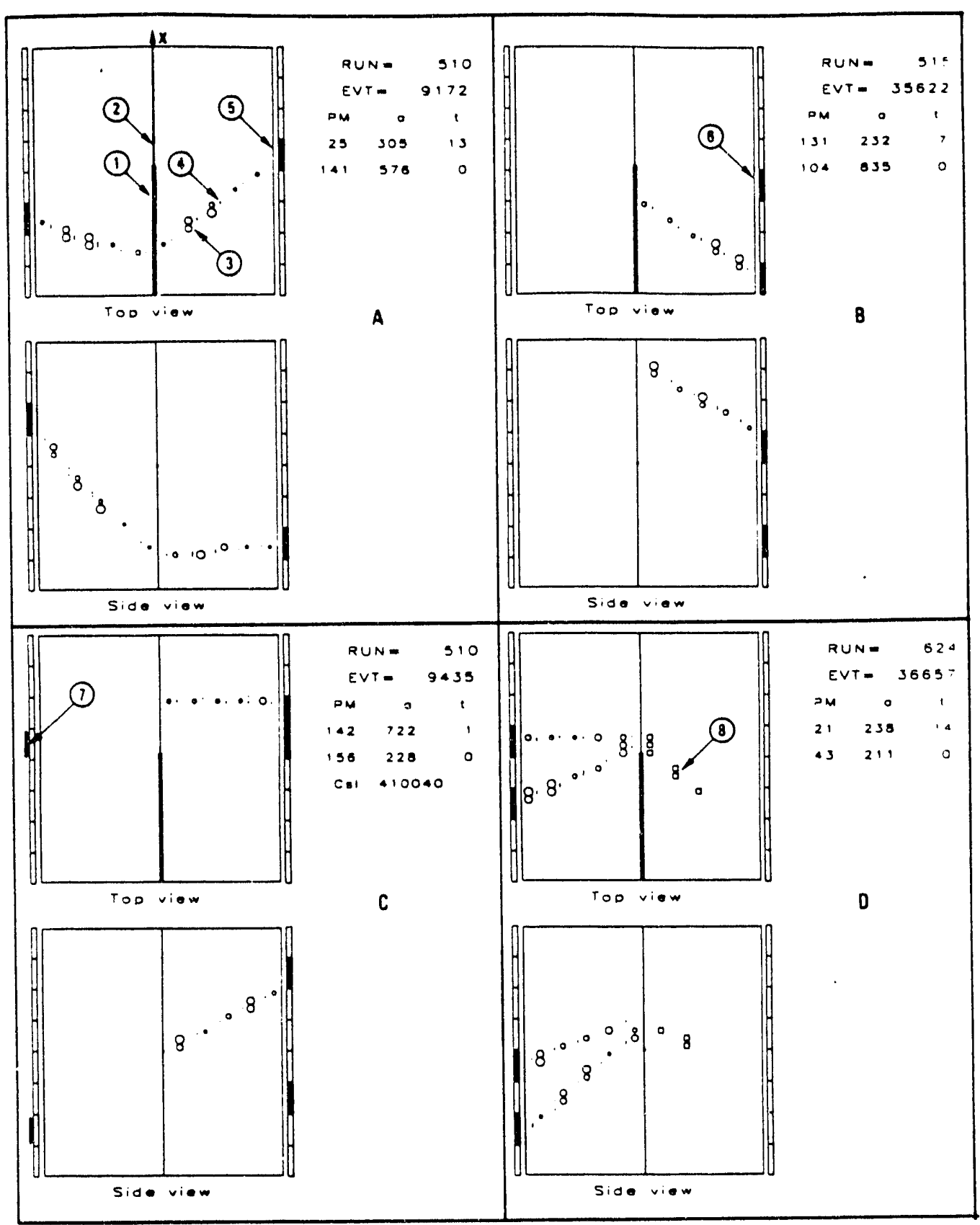

Fig. 2 - Events display

\section{A) e-e event}

1. 100 Mo (the source foil is divided into 2 parts along the $X$ axis)

2 - natural Molybdenum

3- the electron trajectory is tangent to the circles (Geiger cells which are perpendicular to the plane of the figure)

4 - small segments indicate trajectory position along the wire (Geiger cells which are parallel to the plane of the figure)

5 - dark rectangles show the fired plastic counters associated to the tracks. PMTs indications refer to $a=$ energy deposit in $\mathrm{keV}, \mathrm{t}=\mathrm{time}$ in tenth of $\mathrm{ns}$ with respect to the first fired counter.

B) e- $\gamma$ event : the electron track is associated to a fired counter

6 - fired counter corresponding to the detection of a photon

C) $e-\gamma+X$-ray event

7 - the CsI fired signs the X-ray. A $40 \mathrm{keV}$ energy has been reconded.

D) e-e $+\alpha$ event

8 - the squares indicate a delayed short track. Hits are delayed by $98 \mu s$. This is a signature of an $\alpha$ particle. 
As most radioactive decays have small detection efficiencies in the e-e channel, the typical number of Monte Carlo generated events for background sources is of the order of half a million for each nucleus. For the $2 \beta 0 \mathrm{v}$ decay, the e-e channel only has contributing background isotopes from $208 \mathrm{Tl}$ and $214 \mathrm{Bi}$.

Simulations have also been performed for the decays of interest: $2 \beta 2 v$, $2 \beta(0 v, \chi), 2 \beta 0 v$ (ground state and excited states $2_{1}^{+}$and $0_{1}^{+}$).

\section{BACKGROUND STUDIES (AUGUST 91-NOVEMBER 92)}

Between August 91 and February 92, 2240 hours of data were taken with a central source divided into 2 parts, one of high purity copper foil and one of standard natural Molybdenum (Ref. 5,6). The copper foil data show no e-e events beyond 2.5 $\mathrm{MeV}$. Events in the e-e and e- $\gamma$ channels are consistent with the external $\gamma$-flux coming from the photomultipliers (Ref. 7). The standard natural Molybdenum data, using e-e, $e-\gamma$ and $e-\gamma-\alpha$ channels and their respective computed efficiencies give contaminations which accord well with those measured by Germanium spectroscopy.

In a second run, from April 92 to November 92, data were taken for 4640 hours with a high purity Mo foil. The source activity was found to be less than $1 \mathrm{dpm} / \mathrm{kg}$ of ${ }^{214} \mathrm{Bi}$ and $0.1 \mathrm{dpm} / \mathrm{kg}$ of ${ }^{208} \mathrm{Tl}$, again in agreement with the Germaniurn measurements.

Given the low levels of internal activity the rate of events is mainly due to the external flux of photons coming from the photomultiplier tubes. Thus a more precise determination of the source activity is limited by the NEMO 2 detector's activity.

\section{DATA ANALYSIS FOR THE ENRICHED 100Mo SOURCE (DECEMBER 92- MAY 93 )}

The raw data are shown in Fig. 3 and 4 corresponding to a run time of 2485 hours. In the e-e channel, the X, Y position of the vertices in the source plane are presented in Fig. 3a, and the projection on the $X$ axis in Fig. 3 b. The excess of events in the $100 \mathrm{Mo}$ part is clear. To date, the corresponding plots (Fig. $4 \mathrm{a}, 4 \mathrm{~b}$ ) in the e- $\gamma$ channel do not show different behaviors for the enriched $(98.3 \%$ of $100 \mathrm{Mo})$ and natural $(9.6 \%$ of $100 \mathrm{Mo}$ ) Molybdenum foils.

In order to check the existence of beta emitters $\left(234 \mathrm{mPa},{ }^{90} \mathrm{Y}\right)$ in the foils without $\gamma$-ray emission, giving e-e events by Moller scattering, a special one-track trigger was invoked for a short running time (64 hours). In the analysis of these data, the contribution of the external background was minimized by using only events with 


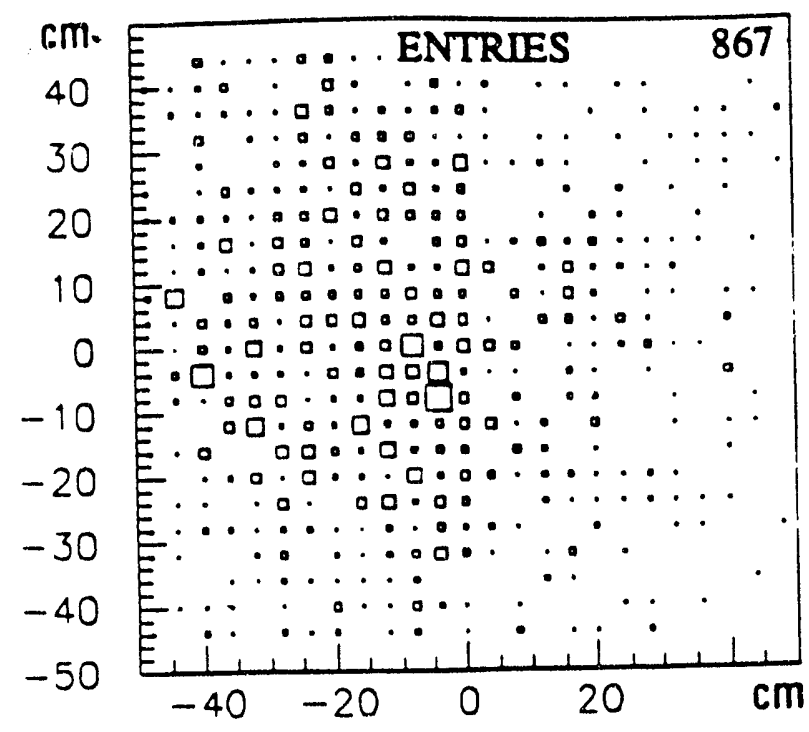

a)

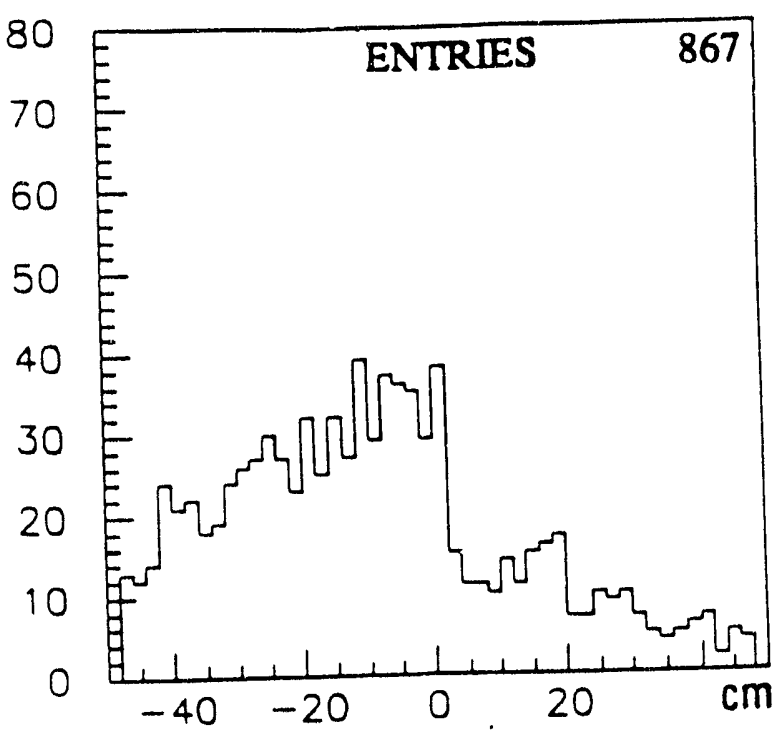

b)

Fig. 3 - Distribution of vertices position for e-e events (867 events)

a) in the X-Y plane of the source foils

b) projection on the $X$ axis

The 100Mo part exhibits an excess of events

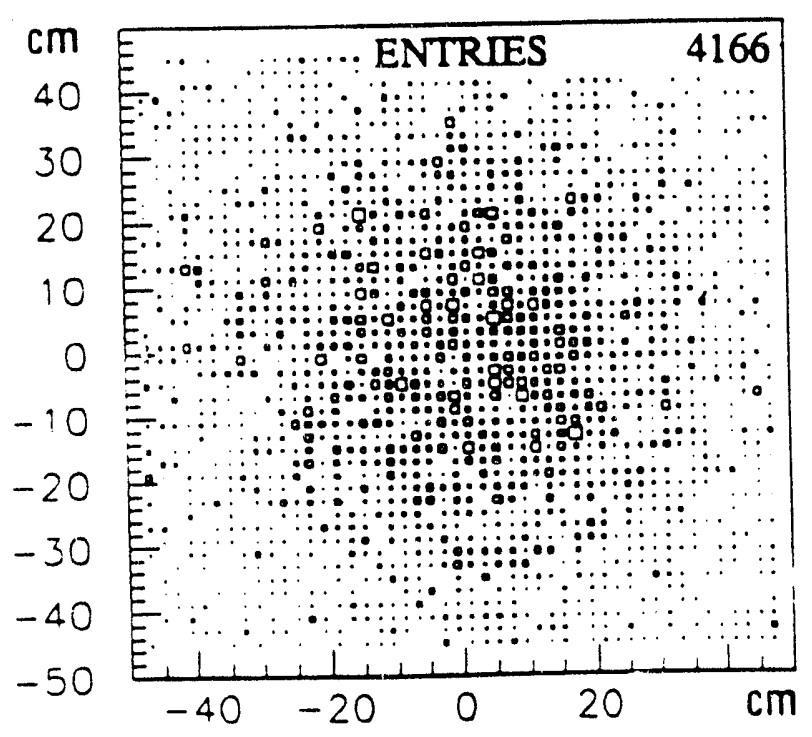

a)

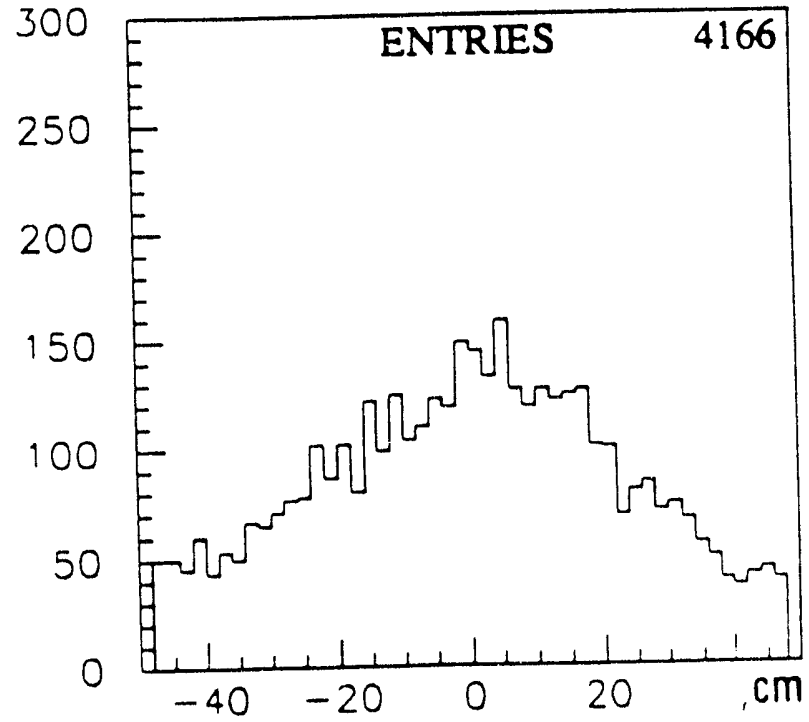

b)

Fig. 4 - Distribution of vertices position for e- $\gamma$ events (4166 events)

a) in the X-Y plane of the source foils

b) projection on the $X$ axis 
an energy deposit greater than $1.4 \mathrm{MeV}$. Fig. 5a presents the $\mathrm{X}$ vertex distribution and. does not show a significant difference between the natural and enriched Molybdenum. The energy spectrum for all selected events (Fig. 5b) can be fitted, in its high energy part, with a maximum contamination of $4 \mathrm{dpm} / \mathrm{kg}$ of beta emitters. From this analysis, it is deduced that the difference of contamination in $234 \mathrm{mPa}$ and $90 \mathrm{Y}$ between natural and enriched Molybdenum is less than $1 \mathrm{dpm} / \mathrm{kg}$ which would correspond to $\leq 10$ events in the e-e channel.

The energy spectra of e-e events for enriched and natural Molybdenum are given in Fig.6. Shapes are different especially in the energy region beyond $1200 \mathrm{keV}$. Currently, no event beyond $2.6 \mathrm{MeV}$ has been recorded.

The analysis of the experimental e- $\gamma$ events and Monte-Carlo simulation, using the $\gamma$ energy spectrum, leads to an upper limit on the ${ }^{214} \mathrm{Bi}$ and ${ }^{208} \mathrm{Tl}$ contamination: $<1 \mathrm{dpm} / \mathrm{kg}$ for ${ }^{214} \mathrm{Bi},<0.1 \mathrm{dpm} / \mathrm{kg}$ for ${ }^{208} \mathrm{Tl}$ in agreement with Germanium measurements. This level of contamination corresponds to a number of e-e events of $\leq 20$ in the whole spectrum.

The external photon flux is mainly due to $40 \mathrm{~K}$ in the PMT glass and yields e-e events by double Compton effect or Compton plus Moller effect on Molybdenum. Most of the events coming from the natural Molybdenum foil are due to such

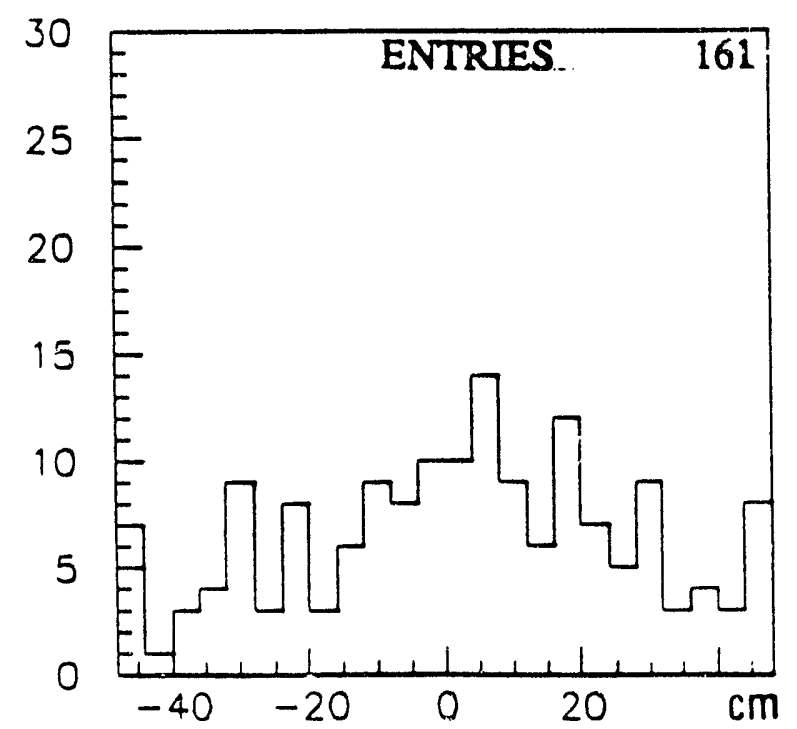

a)

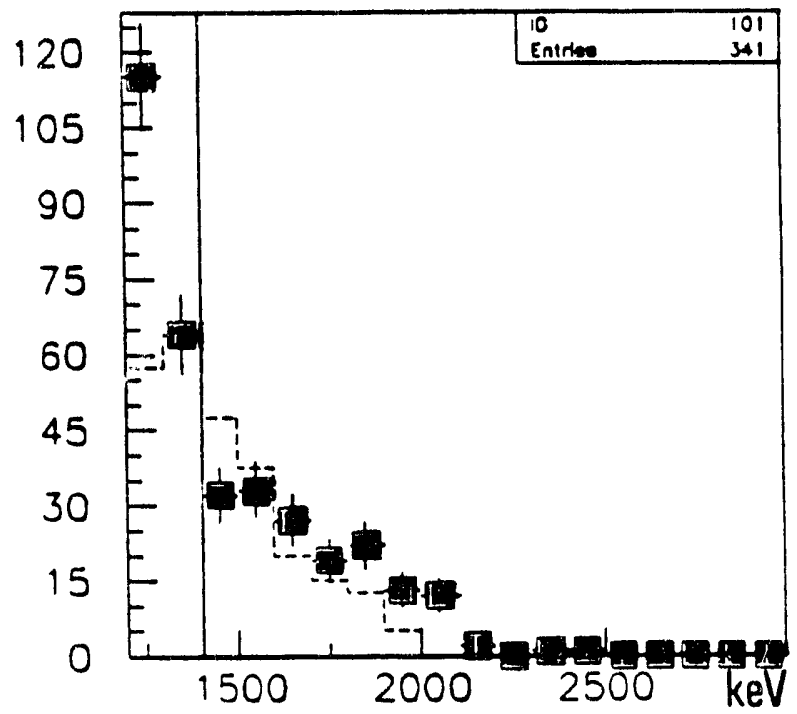

b)

Fig. 5 - One-track trigger events (161 events)

a) $\mathrm{X}$ vertex distribution. Events with energy greater than $1400 \mathrm{keV}$ show similar behavior on enriched and natural Molybdenum

b) experimental energy spectrum (squares). For E $>1400 \mathrm{keV}$ data are compared to a simulated contamination of $4 \mathrm{dpm} / \mathrm{kg}$ (dashed line). 
processes. Events produced by the Compton effect in the scintillator and crossing the tracking volume are rejected by time of flight criteria, and after analysis unrejected events by time of flight measurement are estimated to be less than 10 among the 664 selected events.

The e-e spectrum of $100 \mathrm{Mo}$ after background substraction (Fig. 7) has been fitted by the $2 \beta 2 v$ process $\left(\chi^{2}\right.$ method). The external background in the $100 \mathrm{Mo}$ foil was estimated from the data on natural Molybdenum using a normalization factor deduced from the e- $\gamma$ channel analysis on both foils to take into account differences in masses, thicknesses, etc. The internal background contribution to the e-e spectrum after substraction is negligible. The $2 \beta 2 \nu$ computed efficiency is $\varepsilon=2.0 \%$. From the fit, the deduced half-life is

$$
\mathrm{T}_{1 / 2}=1.0 \pm 0.08 \text { (stat.) } \pm 0.2 \text { (syst.) } 1019 \mathrm{y} \text {. }
$$

Systematics are mainly due to Monte-Carlo efficiencies $(\approx 15 \%)$, background estimations $(\approx 10 \%)$ and selection/tracking of events $(\approx 5 \%)$.

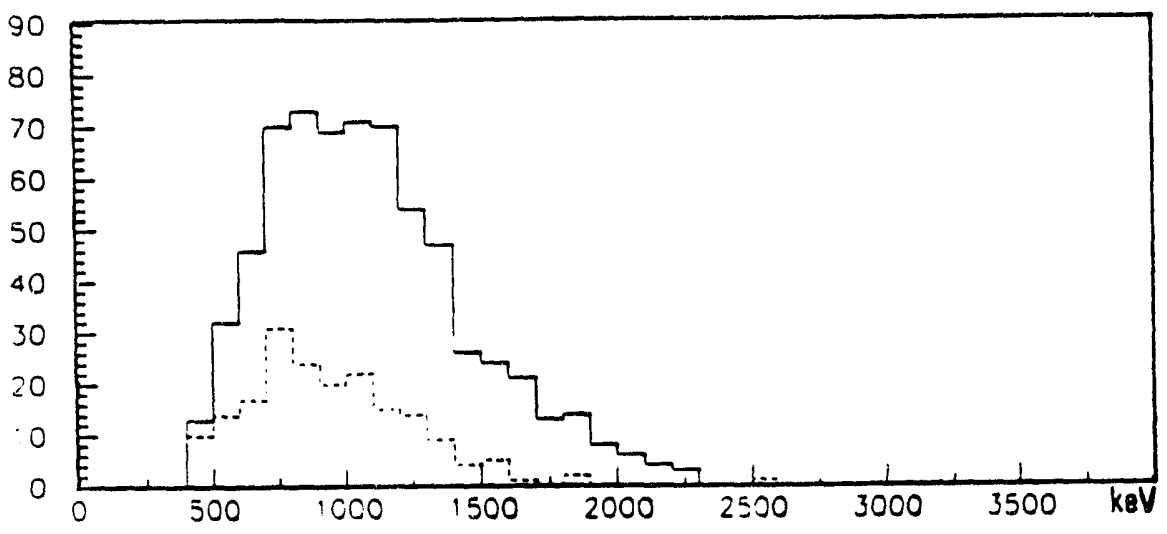

Fig. 6 - Total energy spectrum of e-e events $(2485 \mathrm{~h})$.

Solid line corresponds to $100 \mathrm{Mo}$ (664 events) and dashed ine to natural Molybdenum (189 events). The shapes of the spectra show a different energy behavior.

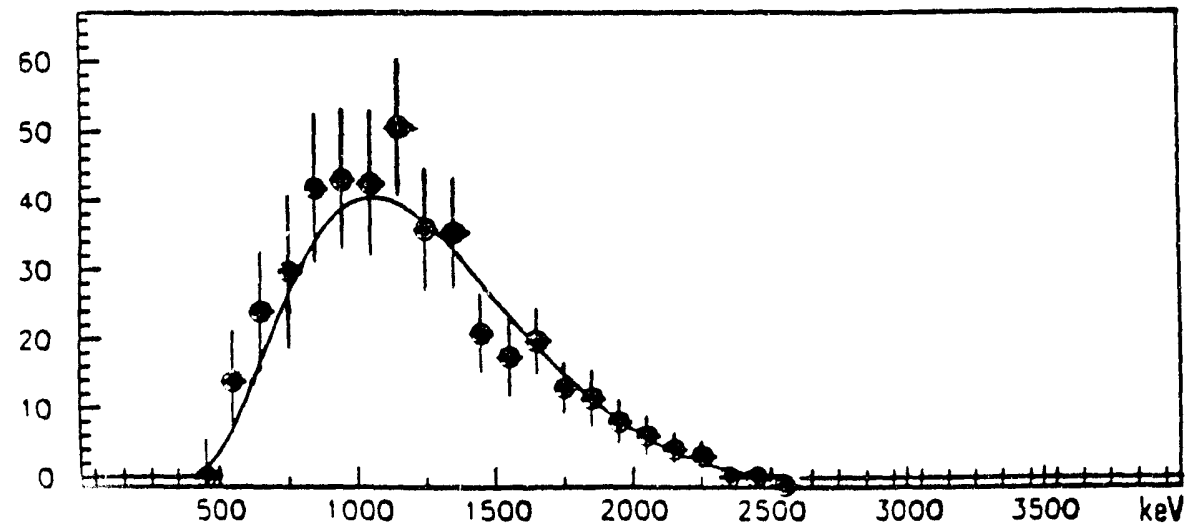

Fig 7 - Total energy spectrum fit for e-e events in $100 \mathrm{Mo}$ (380 events, background subtracted, $2485 \mathrm{~h}$ ). Solid line shows the $2 \beta 2 v$ simulated spectrum fitted to the experimental data. Error bars are only due to statistics. 
Additionally, limits for other processes are given in Table 2 asing Particle Data Group recommendations (Ref. 8)

\begin{tabular}{|c|c|c|c|c|}
\hline decay & $\begin{array}{c}2 \beta 0 v \\
\text { ground state }\end{array}$ & $2 \beta(0 v, \chi)$ & $\begin{array}{c}2 \beta 0 v \\
\text { excited state } 2_{1}^{+}\end{array}$ & $\begin{array}{c}2 \beta 0 v \\
\text { excited state } 0_{1}^{+}\end{array}$ \\
\hline $\begin{array}{c}\text { energy } \\
\text { window } \\
{\left[\mathrm{E}_{1}, \mathrm{E}_{2}\right] \mathrm{MeV}}\end{array}$ & {$[2.5,3.0]$} & {$[2.0,3.0]$} & {$[2.0,2.5]$} & {$[1.4,1.8]$} \\
\hline $\mathrm{N}_{\text {exp }}$ & 0 & 13 & 13 & 98 \\
\hline $\begin{array}{c}\mathrm{N}_{\text {Backgr. }} \\
2 \beta 2 \mathrm{v}+ \\
\text { external back }\end{array}$ & 0. & 14. & 14. & 116. \\
\hline$\varepsilon(\%)$ & 4.5 & 1.8 & 3.5 & 2.9 \\
\hline $\begin{array}{c}\mathrm{T}_{1 / 2} \text { years } \\
(90 \% \mathrm{CL})\end{array}$ & $3.810^{21}$ & $5.010^{20}$ & $9.710^{20}$ & $5.110^{20}$ \\
\hline
\end{tabular}

Table 2 : Half-life limits for $\beta \beta$ decays of $100 \mathrm{Mo}$ to ground and excited states of $100 \mathrm{Ru}$.

\section{CONCLUSION}

Results presented here are preliminary. The experiment will run till October 93 and should improve statistics by a factor of 2 . The measured half-life for $2 \beta 2 v$ decay of ${ }^{100} \mathrm{Mo}$ is in agreement with the previously published results (Ref. 9, 10). In this phase of Research and Development the most important result is the eventless 3 $\mathrm{MeV}$ region, with no e-e event in the total statistics, August 91-May 93. This was achieved with a thorough understanding of the various contributions of background sources. In the near future improvements will be made in the NEMO 2 detector by upgrading it with low radioactive PM tubes. 


\section{REFERENCES}

1- Double beta decay prototype detector with multiwire drift tubes in the Geiger mode. NEMO Collaboration. TAUP 91. Presented by D. Lalanne and Nucl. Phys. B (Proc. Suppl.) 28A (1992) 223-225.

2- Double beta decay prototype detector with multiwire drift tubes in the Geiger mode. NEMO Collaboration. NIM. A 309 (i991) 465-475.

3- Ultralow radioactivity measurements in the Fréjus Underground Laboratory. TAUP 91. J. Busto et al. Presented by F. Hubert and Nucl. Phys. B (Proc. Suppl.) 28A (1992) 425-429.

4- Mesure de la radiopureté en thallium 208 et en bismuth 214 d'une feuille de molybdène avec le détecteur NEMO, F. Natchez, thèse LAL 93-05.

5- The Neutrinoless Experiment with MOlybdenum NEMO 2, NEMO Collaboration. Presented by J. E. Campagne. XXVII Rencontre de Moriond, Les Arcs, Savoie, Jan. 25 - Feb 1, 1992.

6- Neutrinoless Experiment with MOlybdenum, NEMO Collaboration. Presented by S. Jullian. WIEN 92, International Symposium on Weak and Electromagnetic Interactions in Nuclei, Dubna, June 16-22, 1992.

7- Le bruit de fond de radioactivité naturelle dans le détecteur NEMO 2, M. C. Isaac, thèse CENBG, Université de Bordeaux, 1993

8- Particle Data Group. Phys. Rev. D45, 11, part II (1992) III, 40.

9- H. Ejiri et al., Phys. Lett. B258 (1991) 17.

10- S. R. Elliott et al., J. Phys. G. Nucl. Part. Phys. 17 (1991) S145.

\section{DISCLAIMER}

\footnotetext{
This report was prepared as an account of work sponsored by an agency of the United States Gever Government nor any agency thereof, nor any of their Government. Neither the United States Gnvernment nor any agency thereor, nor any or the bility for the accuracy, completeness, or usefulness of any information, apparatus, product, or bility for the accuracy, completeness, or usefulness of any information, apparatus, product, ence herein to any specific commercial product, process, or service by trade name, trademark, ence herein to any specific commercial product, process, or service by tits endorsement, recommentation, or favoring by the United States Government or any agency thereof. The views mendation, or favoring by the United States not necessarily state or reflect those of the United States Government or any agency thereof.
} 

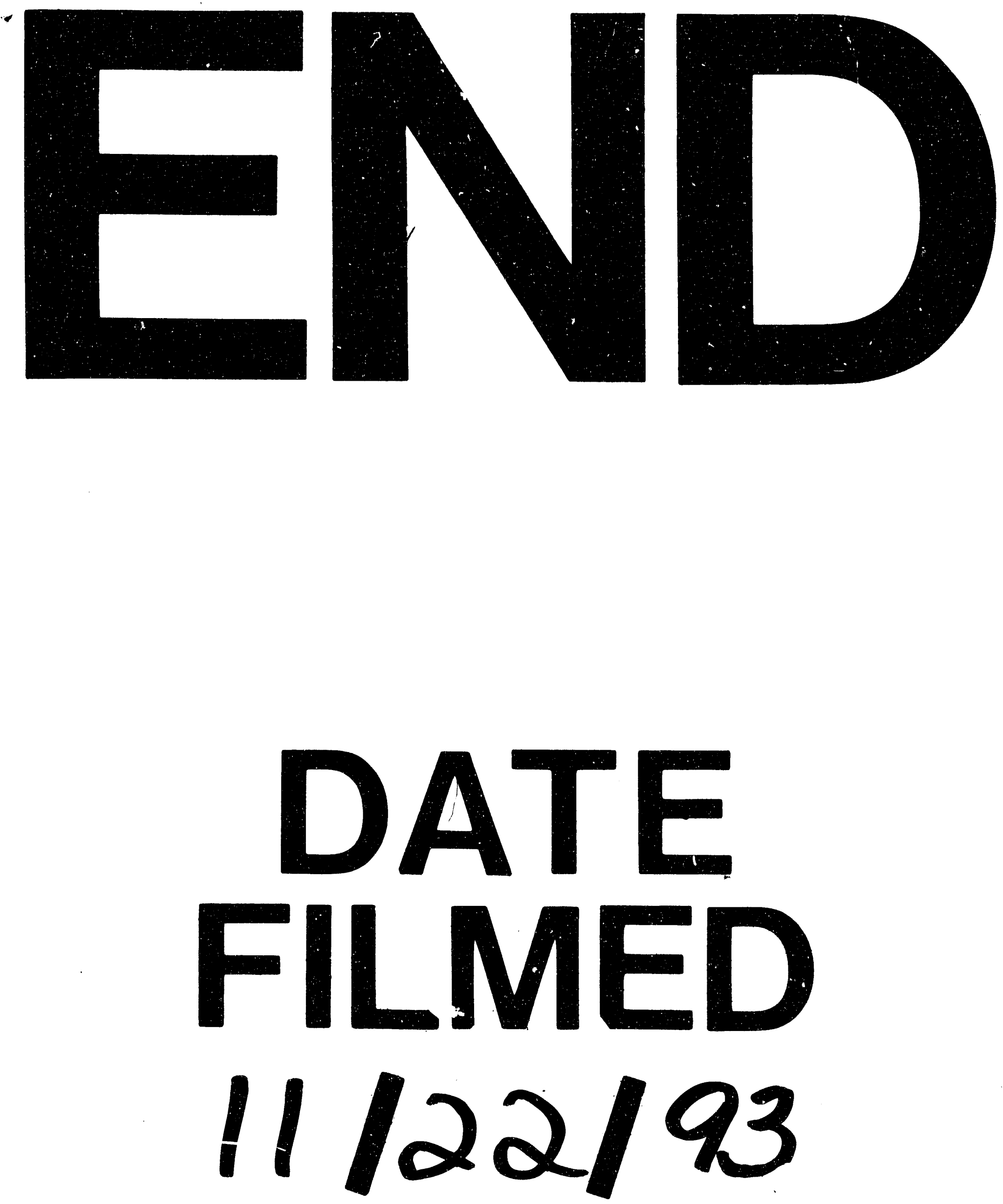